\title{
Congenital Melanocytic Nevi With Meyerson Phenomenon: Two Case Reports and Review of the Literature
}

\author{
Ambra Di Altobrando, ${ }^{1}$ Iria Neri, ${ }^{1}$ Annalisa Patrizi, ${ }^{1}$ Michela Tabanelli, ${ }^{2}$ Cosimo Misciali, ${ }^{1}$ \\ Carlotta Baraldi, ${ }^{1}$ Francesco Savoia ${ }^{1}$
}

\begin{abstract}
1 Department of Experimental, Diagnostic and Specialty Medicine, Dermatology, University of Bologna, Italy
2 Dermatology Unit, AUSL della Romagna, Ravenna, Italy
\end{abstract}

Key words: congenital melanocytic nevus, Meyerson phenomenon, dermoscopy, dermatopathology, topical steroids

Citation: Di Altobrando A, Neri I, Patrizi A, Tabanelli M, Misciali C, Baraldi C, Savoia F. Congenital melanocytic nevi with Meyerson phenomenon: two case reports and review of the literature. Dermatol Pract Concept. 2020;10(3):e2020064. DOI: https://doi.org/10.5826/ dpc.1003a64

Accepted: March 31, 2020; Published: June 29, 2020

Copyright: $\odot 2020$ Di Altobrando et al. This is an open-access article distributed under the terms of the Creative Commons Attribution License, which permits unrestricted use, distribution, and reproduction in any medium, provided the original author and source are credited.

Funding: None.

Competing interests: The authors have no conflicts of interest to disclose.

Authorship: All authors have contributed significantly to this publication. Francesco Savoia and Ambra Di Altobrando equally contributed to the paper.

Corresponding author: Ambra Di Altobrando, MD, Via Massarenti 1, 40138 Bologna, Italy. Email: ambra.dialtobrando@studio.unibo.it

\section{Introduction}

Meyerson phenomenon (MP) is an uncommon condition consisting of a symmetric area of eczema encircling a preexisting central cutaneous lesion such as a melanocytic nevus, a nonmelanocytic skin neoplasia, or a melanoma. MP has been reported to occur both in acquired melanocytic nevi in adults and congenital melanocytic nevi $(\mathrm{CMNi})$ in the pediatric population [1].

\section{Case Presentations}

\section{Case 1}

A 2.5-year-old boy was referred to us for a small CMN of the back encircled by an eczematous patch (Figure 1A). The patient, who had been suffering from atopic dermatitis since birth, complained of itching. Dermoscopy revealed a multi- component pattern, with atypical globules and blotches of pigment, a central blue veil, along with perilesional erythema (Figure 1B). Therapy with a topical mometasone furoate led only to transient improvement, with recurrence after discontinuation and with persistence of the alarming dermoscopic features. Surgical excision of the lesion was performed and histology was suggestive of a CMN with MP. The excision of the lesion led to complete healing.

Case 2

A 6-year-old boy was referred to us for a medium-sized $\mathrm{CMN}$ of the right thigh that had progressively changed over the previous 12 months. On physical examination, a brown patch with ill-defined margins, $6 \mathrm{~cm}$ in maximum diameter, surmounted by yellow crusts and fissures, was observed (Figure 2A). The patient complained of itching and pain. He had suffered from atopic dermatitis during the first years of life, in remission at the time of consultation. Dermoscopic 


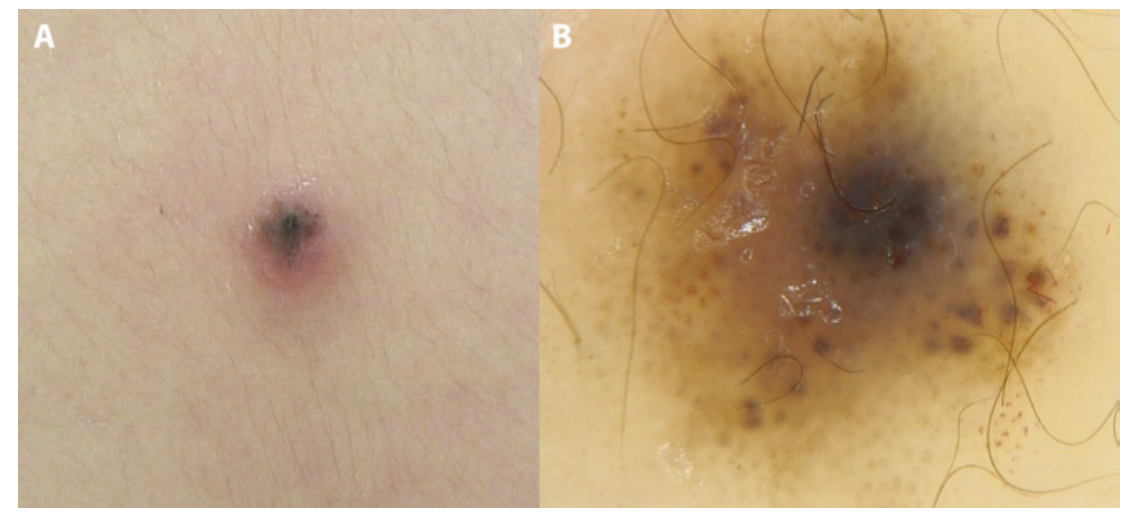

Figure 1. (A) A small congenital melanocytic nevus with Meyerson phenomenon. (B) Dermoscopy revealed a multicomponent pattern, with atypical globules and blotches of pigment, a central blue veil, along with perilesional erythema.

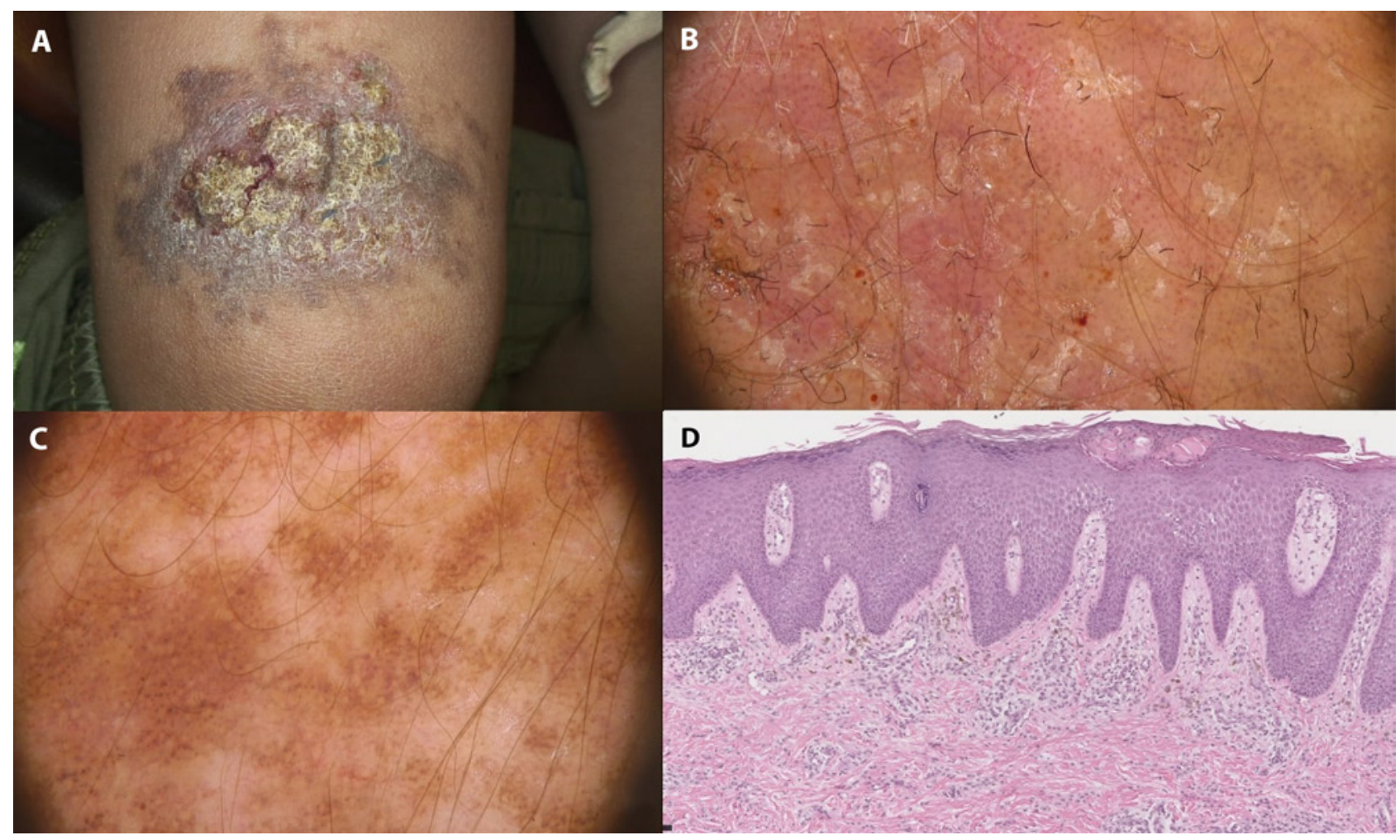

Figure 2. (A) A medium-sized congenital melanocytic nevus surmounted by yellow crusts and fissures. (B) Dermoscopy showed scales, glomerular/dotted vessels, and short linear black fibers stuck to the eczematous surface. (C) Areas with a homogeneous pigmentation were observed on dermoscopy. (D) Histopathology showed the features of a normal congenital melanocytic nevus associated with a chronic dermatitis.

examination revealed areas characterized by pigment network and areas with a homogeneous pigmentation, in association with scales and glomerular and dotted vessels (Figure 2, B and C). Moreover, short, linear black fibers of clothing entrapped in the irregular eczematous surface of the lesion were detected. The use of clobetasol propionate $0.05 \%$ cream led to a reduction of the inflammation, without complete healing of the eczema, which recurred immediately after treatment discontinuation. A punch biopsy was performed and histopathology showed the features of a normal CMN associated with a chronic dermatitis, allowing the diagnosis of CMN with MP (Figure 2D).

Because of the severe pruritus and the chronic course despite therapy, seriate excisions were planned and performed, in order to achieve complete healing.

\section{Discussion}

MP involving CMNi in the pediatric population is rare (Table 1). The most important differential diagnosis is mel- anoma, because the halo of eczema may cause clinical and worrisome dermoscopic features, including "blue-white structures" and "blue areas" [1].

Although MP can have a spontaneous complete resolution when associated with CMN, some authors have reported a chronic course with complete healing achieved only after excision of the CMN [2]. The use of potent topical steroids is associated with faster healing, even though recurrence of MP can occur within the same nevus or in different lesions [2]. 


\begin{tabular}{|c|c|c|c|c|c|c|}
\hline 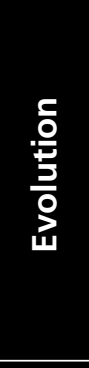 & 1 & 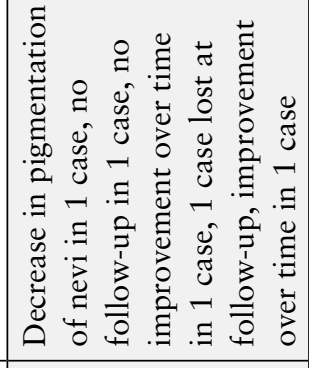 & 1 & 1 & 1 & 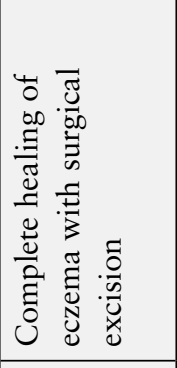 \\
\hline 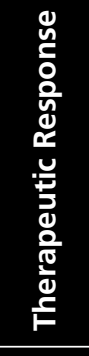 & 1 & 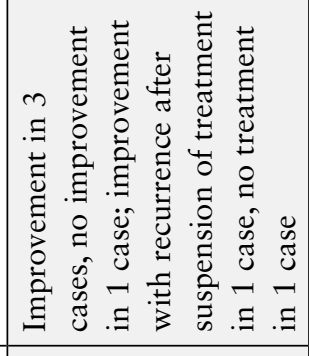 & 1 & 1 & 1 & 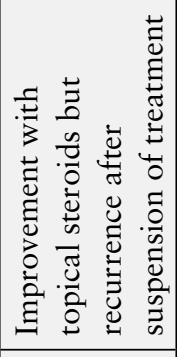 \\
\hline $\begin{array}{l}\frac{\partial}{0} \\
\frac{a}{0} \\
\frac{d}{5} \\
F\end{array}$ & 1 & 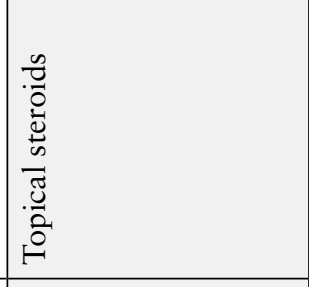 & 1 & 1 & 1 & 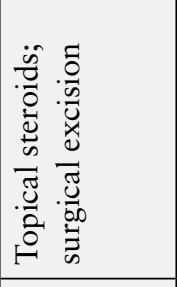 \\
\hline 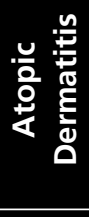 & z & 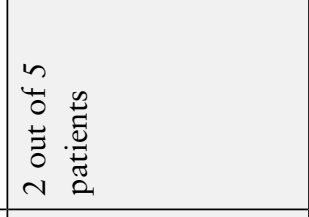 & 1 & 1 & 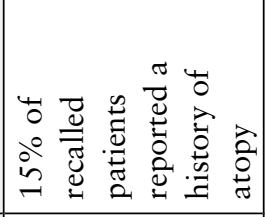 & $\stackrel{0}{\pi}$ \\
\hline 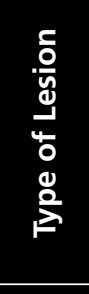 & 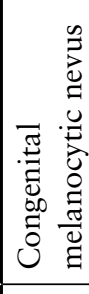 & 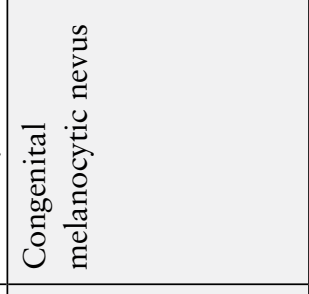 & 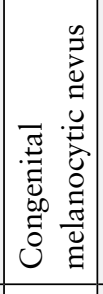 & 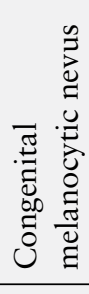 & 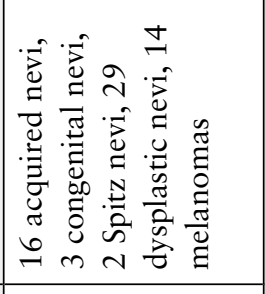 & 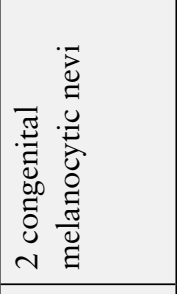 \\
\hline 을 & 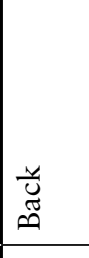 & 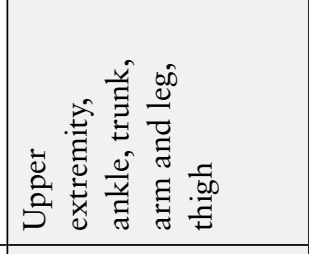 & 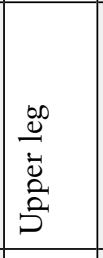 & 毒 & 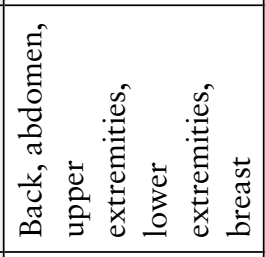 & 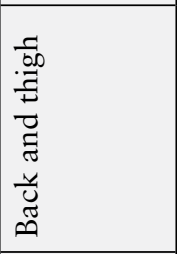 \\
\hline 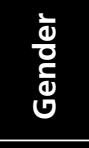 & $\omega_{1}$ & $\begin{array}{l}\omega \\
N \\
\Sigma \\
n \\
m\end{array}$ & $\Sigma$ & $\Sigma$ & 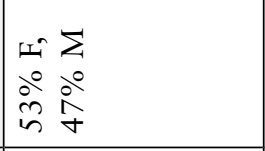 & $\underset{N}{\Sigma}$ \\
\hline ஓ্ & 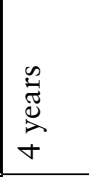 & 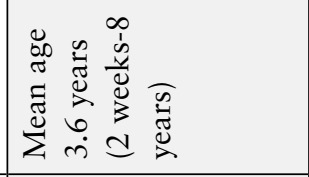 & 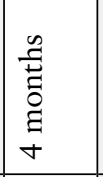 & 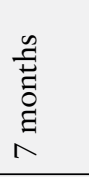 & 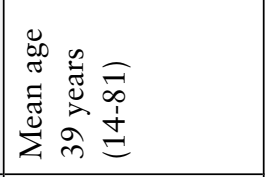 & 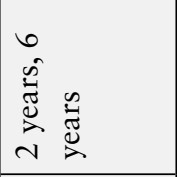 \\
\hline 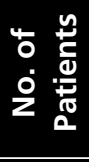 & - & in & - & - & in & N \\
\hline 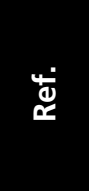 & $\begin{array}{l}\Xi \\
\bar{d} \\
\frac{\bar{v}}{0} \\
2\end{array}$ & 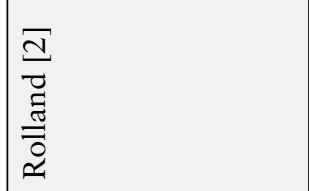 & 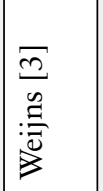 & 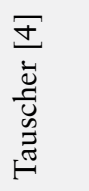 & 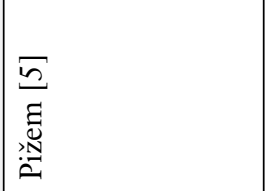 & 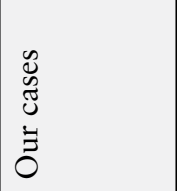 \\
\hline
\end{tabular}

\section{Conclusions}

Our cases highlight the fact that in children MP in CMNi can have a chronic course, with severe eczema, itch, and pain, and can be responsible for alarming dermoscopic features, simulating a melanoma.

\section{References}

1. Richey P, Radfar A, Kirkorian AY. Blue areas on dermoscopy of a congenital nevus with Meyerson phenomenon. J Am Acad Dermatol. 2015;73(5):e161-e163. https:// doi.dx.org/10.1016/j.jaad.2015.07.004.

2. Rolland S, Kokta V, Marcoux D. Meyerson phenomenon in children: observation in five cases of congenital melanocytic nevi. Pediatr Dermatol. 2009;26(3):292297. https://doi.dx.org/10.1111/j.15251470.2009.00931.x.

3. Weijns ME, Houtappel M. An infant with a nevus on the upper leg [in Dutch]. Ned Tijdschr Geneeskd. 2017;161:D1532. PMID: 28880143.

4. Tauscher A, Burch JM. Picture of the month-quiz case. Diagnosis: Meyerson phenomenon within a congenital melanocytic nevus. Arch Pediatr Adolesc Med. 2007;161(5):471-472. https://doi. dx.org/10.1001/archpedi.161.5.471

5. Pižem J, Stojanovič L, Luzar B. Melanocytic lesions with eczematous reaction (Meyerson's phenomenon)—a histopathologic analysis of 64 cases. J Cutan Pathol. 2012;39(10):901-910. https://doi.dx. org/10.1111/j.1600-0560.2012.01960.x. 